Thomas Pölzler

\title{
Comment on Sabine Hohl and Dominic Roser. Stepping in for the Polluters? Climate Justice under Partial Compliance
}

\section{Introduction}

The distinction between ideal and non-ideal theorizing has been drawn in a variety of different ways. One way of construing the distinction is in terms of fact-sensitivity (Farelly 2007, 844). According to extreme ideal theory, moral prescriptions are not contingent on empirical facts. What ought to be is completely independent of what is. Extreme non-ideal theory, on the other hand, is based on the assumption that moral prescriptions are contingent on all sorts of empirical facts (e.g., facts about compliance, historical conditions, institutional design, or human nature).

Construed in this way, ideal and non-ideal theory turn out to be points on a spectrum rather than mutually exclusive positions. Between the sketched extreme variants are innumerable further possible positions. The approach taken by Sabine Hohl and Dominic Roser in their article "Stepping in for the Polluters? Climate Justice under Partial Compliance" exemplifies such a mediating (rather non-ideal) position. States' duties with regard to climate change are taken to be contingent on the empirical fact that states do not fully comply with their duties, but not on various other empirical facts.

The result of Hohl and Roser's analysis is that states that emit their fair share of greenhouse gases (compliers) may have a duty to step in for states that emit more than their fair share (non-compliers). They might have a duty to emit less than their fair share, or to take additional action against dangerous climate change of some other kind (498).

This conclusion may seem surprising. Isn't it unfair that those who already comply have to shoulder additional efforts because others refuse to do what they should do? However, Hohl and Roser are quite convincing in making their case. My goal in this comment, therefore, is not to examine any of Hohl and Roser's arguments in particular, but rather to discuss two related issues. First, I will ask how relevant the duty of states to step in for the polluters is in practice 
(given the degree of 'ideality' of Hohl and Roser's approach). Second, I will ask whether there is such a duty on more non-ideal approaches as well.

\section{How Relevant is the Duty to Step in for the Polluters?}

Although I agree with Hohl and Roser that it is possible for states to be under a duty to take up the slack (given the degree of 'ideality' of their approach), I do not believe that this duty is very relevant in practice. All things considered, few states will turn out to be under a duty to take up the slack. Those states that will turn out to be under such a duty will have to take up only a small proportion of the slack.

In their article, Hohl and Roser mention a number of factors that limit the extent of states' duty to take up the slack. In what follows I will look at three of these factors.

\subsection{Demandingness}

One condition for being under a duty to take up the slack is that taking up the slack is not overdemanding. If a state cannot bear the costs associated with taking up the slack, a respective duty cannot be ascribed to it. Like Hohl and Roser (482), I suspect that at least some states are sufficiently wealthy to be able to bear the costs associated with taking up the slack (e.g. Switzerland, or Germany). It is important to note, however, that these countries can be under a duty to take up the slack only if they are compliers. Non-compliers can, per definitionem, not be under a duty to take up the slack. They are the problem, not part of the solution. What we have to ask, then, is not only whether there are states that can bear the costs associated with taking up the slack, but whether there are states that can bear these costs and are compliers. This supplement very likely leads to a further reduction of the (already limited) number of potential slack-takers. On any plausible principle of emission distribution, at least some of the sufficiently wealthy nations can be expected not to comply. One reason for this is that states are poorly motivated to comply in general (see below). Another reason concerns wealthy nations in particular. Wealthy nations typically emit much more per capita than poor ones. Wealthy nations also typically emitted much more in the past, and benefited from these emissions. Thus on any plausible principle of distribution many wealthy nations will have to drastically cut their emissions. At least some wealthy nations can be expected to be reluctant to bear the high costs associated with such drastic reductions (despite their being able to afford them).

That at least some sufficiently wealthy nations will not comply is also suggested by a look at the Kyoto Protocol. Nations like Norway, Australia, New Zealand, the Netherlands, Austria, and Canada fail spectacularly in meeting their emission reduction targets (International Energy Agency 2011, 13). The latter even withdrew from the treaty some months ago. Thus, considerations 
about overdemandingness give reason to assume that only a very small number of states qualify as slack-takers.

In addition, considerations about overdemandingness also suggest that those states which qualify as slack-takers will have to take up only a small proportion of the slack. It can be assumed that on any plausible principle of emission distribution the number of non-compliers will be considerable (think, again, of the Kyoto Protocol, and see the section on motivation below), and some of the non-compliers will be big emitters (think of the US, the world's second largest emitter, which did not even ratify the protocol). The slack waiting to be taken up will be huge. In fact, it will probably be so huge that the few states qualifying as slack-takers will not be able to bear the costs of taking up all of the slack. ${ }^{1}$ Even taking up half, or a quarter of the slack (say) may prove to be too demanding. If a state is obliged to take up the slack, it will probably be required to take up only a small proportion of it.

\subsection{Fairness}

Hohl and Roser concede that having to take up the slack is unfair (482). That said, they assume that the unfairness of having to take up the slack may be outweighed by its positive consequences. Taking up the slack (emitting below one's fair share, or taking additional action against dangerous climate change of some other kind) is thought to prevent infringements of the human rights of future persons. It is thought to prevent future persons from suffering and dying. This consequence is of such great moral importance that it might outweigh the unfairness of having to take up the slack (484). That it in fact does outweigh this unfairness in a given situation is a further condition for a state's having a duty to take up the slack.

When does preventing the infringements of the human rights of future persons outweigh the unfairness of having to take up the slack? As Hohl and Roser (488) note, it is very hard to define a non-arbitrary cut-off point for the duty to take up the slack. Preventing infringements of the human rights of future persons is certainly a goal as morally important as it can get. Nevertheless, I suspect that there may well be situations in which the unfairness of having to take up the slack weighs heavier than the good it does to future persons. Conditions that privilege considerations of unfairness include: high costs of taking up the slack (Horton 2011, 93); little effectiveness of taking up the slack in preventing infringements of the human rights of future persons; non-compliers that could comply at low costs; non-compliers that could bear the costs of complying more easily than slack-takers could bear the costs of taking up the slack; or malicious non-compliers, i.e., non-compliers whose main reason for not complying is to impose the costs of taking up the slack on the compliers. Factors such as these increase the likelihood that a complier will be exempt from the duty to take up the slack, or will be obliged to take up only a small part of the slack. My guess is that in fact at least some of the above factors will often be present to some

\footnotetext{
1 It might even be doubted whether it is possible for those nations to take up all of the slack (478, footnote 1$)$.
} 
degree, in particular the factors of high costs of taking up the slack, and noncompliers that could bear the costs of complying more easily than slack-takers could bear the costs of taking up the slack. This means that like considerations about demandingness, considerations about fairness are likely to impose narrow limits on the practical relevance of states' duty to take up the slack.

\subsection{Effectiveness}

The outcome of the trade-off between benefiting future persons and considerations of fairness depends, to a significant degree, on how effective taking up the slack is in preventing infringements of the human rights of future persons. How many future persons can be protected from human rights violations by taking up a given part of the slack? How serious are the infringements that can be prevented?

Another crucial condition for a state's being under a duty to take up the slack according to Hohl and Roser is that taking up the slack is effective in preventing infringements of the human rights of future persons at all. If taking up the slack does not prevent such infringements in any way, or if it, on the contrary, even promotes them, then states cannot be under a duty to take up the slack.

In their article, Hohl and Roser discuss two objections against the claim that taking up the slack is effective (488-98). According to the first objection, taking up the slack does not prevent infringements of the human rights of future persons in any way (because it makes no difference as to mitigating climate change). According to the second objection, taking up the slack does not only fail in preventing infringements of the human rights of future persons, but even promotes them (due to feedback effects). I find Hohl and Roser's response to the first objection convincing. Probably the more pressing worry is that taking up the slack creates incentives for free-riding and thus has, all things considered, a negative effect on protecting future persons' human rights. Observing states taking up the slack, compliers might think to themselves: Why shouldn't I emit more than my fair share, if these other states step in for me and emit less? Noncompliers might think to themselves: Why shouldn't I increase my emissions further, if I can rely on these other states' reducing theirs? This might lead to a situation in which, despite some states' taking up the slack, more greenhouse gases are emitted overall, and more future persons are hurt more severely in terms of violations of their human rights (495).

The above worry must certainly be taken seriously. Hohl and Roser plausibly show, however, that it is far from obvious that taking up the slack has the described psychological feedback effects. First, taking up the slack in the form of reducing emissions below one's fair share does not necessarily create strong incentives for other states to decrease their own level of compliance (it can be done secretly, communicated in ways that minimize undesired feedback effects, etc.). Second, the slack might be taken up in forms other than reducing emissions below one's fair share, e.g., by taking adaptive measures or political action. These other forms of taking up the sack might not result in the described psychological feedback effects, or only in weaker such effects (495-498). 
As Hohl and Roser (495-6) note, to assess the effectiveness of taking up the slack would be extremely difficult. It would require surveying not only psychological, but also economic, political and other kinds of feedback effects. The responses of states to a complier's taking up the slack will vary over time, and there may be significant differences between cultures. Thus, with regard to the question of practical relevance, it can only be said that considerations about effectiveness may possibly lead to further decreases, not that they are likely to do so.

The result of the above rough analysis is that at the least considerations about demandingness and fairness put narrow limits to the practical relevance of states' duty to take up the slack. In practice, this duty will apply to only a few states, and those states that will turn out to be under such a duty will have to take up only a small proportion of the slack

\section{Is There a Duty to Step in for the Polluters on More Non-ideal Approaches as Well?}

Hohl and Roser's approach to climate justice is sensitive to the fact that states do not fully comply with their duties of justice, but not to various other empirical facts. Up to now I have assumed that this approach is appropriate. In what follows, I will ask whether states have a duty to take up the slack on more extremely non-ideal approaches as well. Since the number of possible more nonideal approaches is almost unlimited, I will not discuss any particular example of such an approach. Instead, I will focus on two kinds of empirical facts: facts about states' resources for primarily moral goals, and facts about human nature, or, more precisely, human motivation. I will suggest that on any approach that construes morality as contingent on at least one of those facts, there exists either only a limited duty to take up the slack, or no such duty at all.

\subsection{Resources}

Hohl and Roser assume that states have a duty to mitigate climate change because climate change has a realistic potential to infringe on the human rights of future persons (478). However, climate change is not the only phenomenon that actually or potentially infringes on the human rights of persons. Global pandemics or economic collapses have the potential to violate the human rights of future persons as well. Poverty, racial discrimination, gender discrimination and many other phenomena actually violate the human rights of present persons. States do not have the resources to prevent all of these violations of human rights. They are, at least to some degree, forced to trade-off their primarily moral goals against each other.

Taking into consideration that states have limited resources to prevent human rights violations puts limits on a state's duty to take up the slack. In order not to exceed their fair share of emissions, many states (in particular wealthy ones) will have to spend a significant proportion of their moral resources. Let us assume 
that the proportion is justified. Preventing dangerous climate change is given much, but not too much weight in relation to other primarily moral goals. A state's duty to take up the slack threatens to upset this balance. It forces a state to spend even more on preventing dangerous climate change, and thus less on fighting poverty, discrimination, and so on. Assuming that the slack waiting to be taken up is huge (see above), it forces a state to spend even much more on the prevention of dangerous climate change, and thus much less on its other primarily moral goals. But of course, at some point this unilateral fixation will become morally unjustifiable. No state can be required to spend 80 or $90 \%$ of its moral resources on mitigating climate change, while almost completely neglecting present suffering. If morality is construed as contingent on the fact that states have limited resources for primarily moral goals, then the duty to take up the slack turns out to be limited. States do not have a duty to take up all of the slack, but only as much of it as is justified in relation to how much these states spend on other primarily moral goals (which might even mean that a state does not have to take up any part of the slack at all).

\subsection{Motivation}

Morality is often considered to be contingent on facts about motivation. In order for a moral norm to be valid, it is assumed, the addressees of the norm have to be motivated to act in conformity with it to at least some degree (e.g. Carens 1996, 158).

Can states be expected to be motivated to act in conformity with a duty to take up the slack? At best, I believe, the motivation will be very low. Taking up the slack is a duty states primarily have toward persons in the distant future. However, the motivation to prevent the suffering of distant future persons is typically considerably lower than the motivation to prevent the suffering of present persons. One reason for this is that victims in the distant future hardly manage to trigger our emotions. We feel compassion toward the child pinching its finger right in front of us, and at least some compassion toward people starving in Africa (who are given a face by the media). But we feel hardly any compassion toward people drowning in a flood or dying of thirst three hundred years from now (Birnbacher 2008, 29-30; 2009, 282-283). Another reason for the lack of motivation is the "asymmetry of power" (Meyer 2008) between present and distant future persons. We can benefit or harm distant future persons significantly. They, however, cannot do the same to us. They cannot reward us in any significant ways if we prevent them from suffering, nor can they sanction us in any significant ways if we don't (Birnbacher 2008, 30; 2009, 283-284).

In the case of climate change, our motivation is also lowered by various kinds of uncertainties. What if global climate doesn't really change as severely as predicted? What if, on the other hand, it changes so severely that humanity is doomed whatever we do today? In order to make significant differences, subsequent generations need to continue our efforts. Will they do so? What about new technologies? Future generations may perhaps develop new and very effective means of mitigating or adapting to climate change-means that allow 
them to achieve at low cost what we at present can achieve only at very high cost, and so on (e.g. Birnbacher 2009, 284).

Also, taking action against climate change is costly. It requires changing one's habits, one's lifestyle, and it requires spending money.

It is natural to assume that the resulting lack of motivation on the individual level transfers to the level of the state. If this is true, states can be expected to be poorly motivated to take action against climate change. ${ }^{2}$ They can be expected to be poorly motivated not to exceed their fair share of greenhouse gas emissions or to develop or sign treaties that would bind them to do so.

When it comes to taking up the slack, things get even worse, however. States' motivation is lowered further by at least two important factors. First, there is the impression that taking up the slack is unfair. As Hohl and Roser (478; 482-3) point out, states are very concerned with their positions relative to each other. Nobody wants to be 'taken in'. Nobody wants to lose ground in the global competition. It is thus hard to believe that many states would accept being put in a worse position by taking up the slack. ${ }^{3}$ Furthermore, taking up the slack adds another important kind of uncertainty to the picture. As explained above, it is very hard to assess the various possible feedback effects of taking up the slack (e.g. how other states will respond). If it is uncertain whether taking up the slack is effective in preventing infringements of the human rights of future persons at all, however, why should we as a state shoulder this burden?

The problems of unfairness and uncertainty very likely lower states' motivation to take up the slack to a point where it is practically nonexistent. Thus, if morality is construed as contingent on motivation, there may not be such a thing as a duty to take up the slack at all.

\section{Concluding Remarks}

In this comment I have attempted to show two things. First, states' duty to take up the slack is not very relevant in practice (because of considerations of demandingness and fairness). Second, given a more non-ideal approach than the one taken by Hohl and Roser there may be only a weak duty to take up the slack, or no such duty at all (because the duty must be weighed against other duties, and because states are poorly motivated to act in conformity with it).

Concerning the first claim, note that I do not wish to deny that thinking about states' duties to step in for the polluters is relevant. Hohl and Roser's paper is original and stimulating, and may well mark the beginning of a broader discussion.

Concerning the second claim, I only wish to point out that if one takes a more non-ideal approach to climate justice, then there may be only a weak duty

\footnotetext{
${ }^{2}$ When I speak of states being motivated this is not meant to imply that states can actually have motives. It's merely shorthand for the motives of states' representatives and citizens.

3 This is also suggested by psychological findings. Perceptions of unfairness typically provoke anger and spiteful reactions. E.g., in ultimatum game experiments participants are often willing to accept losses if this allows them to sanction unfair distributions by others (e.g. Pillutla/Murnighan 1996).
} 
to step in for the polluters, or no such duty at all. I do not actually advocate taking a more non-ideal approach. The question of which degree of 'ideality' is right goes far beyond this short comment. Moreover, it is not even clear that there is such a thing as a single right approach to questions of morality. It has been suggested that for some purposes (e.g., evaluating certain institutions or practices, establishing long-term goals) more ideal approaches are 'right', whereas for other purposes (figuring out what to do in the here and now) it is more appropriate to adopt a non-ideal perspective (e.g. Carens 1996, 168-169; Mason 2004, 265-267).

\section{Bibliography}

Birnbacher, D. (2008), Langzeitverantwortung - das Problem der Motivation, in: Gethmann, C. F./J. Mittelstraß (eds.), Langzeitverantwortung. Ethik, Technik, Ökologie, Darmstadt, 23-39

- (2009), What Motivates Us to Care for the (Distant) Future?, in: Gosseries, A./L. H. Meyer (eds.), Intergenerational Justice, Oxford, 273-300

Carens, J. H. (1996), Realistic and Idealistic Approaches to the Ethics of Migration, in: International Migration Review 30, 156-170

Farrelly, C. (2007), Justice in Ideal Theory: A Refutation, in: Political Studies 55, 844-864

Hohl, S./D. Roser (2011), Stepping in for the Polluters? Climate Justice under Partial Compliance, in: Analyse $\mathcal{E}$ Kritik 33(2), 477-500

Horton, K. (2011), Fairness and Fair Shares, in: Utilitas 23, 88-93

International Energy Agency (2011), CO2 Emissions From Fuel Combustion-Highlights, Paris, URL: http://www.iea.org/co2highlights/CO2highlights.pdf

Mason, A. (2004), Just Constraints, in: British Journal of Political Science 34, 251268

Meyer, L. (2008), Intergenerational Justice, in: Zalta, E. N. (ed.), The Stanford Encyclopedia of Philosophy, URL: http://plato.stanford.edu/entries/justice-intergenerational/

Pillutla, M./Murnighan, K. (1996), Unfairness, Anger, and Spite: Emotional Rejections of Ultimatum Offers, in: Organizational Behavior and Human Decision Processes $68(3), 208-224$ 